\title{
ARTICLE
}

Molecular Diagnostics

\section{Identification of a biomarker panel for improvement of prostate cancer diagnosis by volatile metabolic profiling of urine}

\author{
Ana Rita Lima $\mathbb{D}^{1}$, Joana Pinto $\mathbb{D}^{1}$, Ana Isabel Azevedo ${ }^{1}$, Daniela Barros-Silva $\mathbb{D}^{2}$, Carmen Jerónimo $\mathbb{D}^{2,3}$, Rui Henrique $\mathbb{D}^{2,3,4}$, \\ Maria de Lourdes Bastos $\mathbb{D}^{1}$, Paula Guedes de Pinho ${ }^{1}{ }^{1}$ and Márcia Carvalho $\mathbb{D}^{1,5}$
}

BACKGROUND: The lack of sensitive and specific biomarkers for the early detection of prostate cancer (PCa) is a major hurdle to improve patient management.

METHODS: A metabolomics approach based on GC-MS was used to investigate the performance of volatile organic compounds (VOCs) in general and, more specifically, volatile carbonyl compounds (VCCs) present in urine as potential markers for PCa detection.

RESULTS: Results showed that PCa patients $(n=40)$ can be differentiated from cancer-free subjects $(n=42)$ based on their urinary volatile profile in both VOCs and VCCs models, unveiling significant differences in the levels of several metabolites. The models constructed were further validated using an external validation set ( $n=18 \mathrm{PCa}$ and $n=18$ controls) to evaluate sensitivity, specificity and accuracy of the urinary volatile profile to discriminate PCa from controls. The VOCs model disclosed $78 \%$ sensitivity, $94 \%$ specificity and $86 \%$ accuracy, whereas the VCCs model achieved the same sensitivity, a specificity of $100 \%$ and an accuracy of $89 \%$. Our findings unveil a panel of 6 volatile compounds significantly altered in PCa patients' urine samples that was able to identify PCa, with a sensitivity of $89 \%$, specificity of $83 \%$, and accuracy of $86 \%$.

CONCLUSIONS: It is disclosed a biomarker panel with potential to be used as a non-invasive diagnostic tool for PCa.

British Journal of Cancer (2019) 121:857-868; https://doi.org/10.1038/s41416-019-0585-4

\section{BACKGROUND}

Prostate cancer (PCa) ranks second in cancer incidence and fifth in mortality among men worldwide. ${ }^{1}$ Diagnostic strategies currently available for patients with PCa rely on prostate biopsy (PB), which is an invasive, unpleasant and potentially harmful procedure, potentially missing clinically significant cancers due to tumour heterogeneity. ${ }^{2}$ Prostate cancer detection based on serum PSA with a cut-off of $4.0 \mathrm{ng} / \mathrm{ml}$ has limited sensitivity (of 20.5\%) and specificity (ranging from 51 to $91 \%$ ), 3,4 and inability to differentiate aggressive from indolent $\mathrm{PCa}_{1}{ }^{4}$ leading to false negatives, to overdiagnosis and consequent overtreatment. ${ }^{5}$ The free/total serum PSA ratio (fPSA/tPSA) has been proposed as an alternative. However, it displays the opposite performance, with high sensitivity but low specificity. ${ }^{3}$ Globally, this entails the performance of a large number of prostate biopsies, a significant proportion of which is deemed unnecessary. Thus, the free/total PSA ratio is not usually employed for risk-stratification of prostate cancer, but only in selected cases. The reported values for the sensitivity and specificity of this biomarker are very inconsistent among different studies, nevertheless a recent meta-analysis concluded that this biomarker shows a sensitivity of $70 \%$ and a specificity of $58 \%{ }^{6}$ Thus, intense efforts have been devoted for development of PCa molecular biomarkers, some of which have already obtained FDA approval, like prostate cancer antigen 3 $(\mathrm{PCA} 3)^{7}$ or circulating tumour cells (CTs). ${ }^{7}$ Notwithstanding, these biomarkers also have important limitations, such as the definition of a cut-off value (e.g., PCA3) ${ }^{7}$ and low abundance at early stages (e.g., CTs). ${ }^{7}$ Thus, discovery and validation of novel PCa biomarkers with improved sensitivity, non-invasive and able to detect earlystage disease (when PCa is potentially curable) remains an important research aim.

Metabolomics emerged as one of the most promising approaches for discovery of new disease biomarkers as pathological conditions cause disruption of metabolic processes and consequently change the production, use and levels of many metabolites, resulting in a characteristic "metabolic signature" that can be captured through metabolic profiling. Analysis of the volatile part of the metabolome, i.e. the low molecular weight volatile organic compounds (VOCs) present in the headspace (gas phase) of clinical samples (e.g., biofluids as urine), is a promising new screening tool for several cancers, including PCa. ${ }^{8-10}$ VOCs are end products of cellular activities and alterations in VOCs

\footnotetext{
${ }^{1}$ UCIBIO/REQUIMTE, Department of Biological Sciences, Laboratory of Toxicology, Faculty of Pharmacy, University of Porto, Porto, Portugal; ${ }^{2}$ Cancer Biology \& Epigenetics Group, Research Center (CI-IPOP) Portuguese Oncology Institute of Porto (IPO Porto), Porto, Portugal; ${ }^{3}$ Department of Pathology and Molecular Immunology-Biomedical Sciences Institute (ICBAS), University of Porto, Porto, Portugal; ${ }^{4}$ Department of Pathology, Portuguese Oncology Institute of Porto (IPO Porto), Porto, Portugal and ${ }^{5}$ UFP Energy, Environment and Health Research Unit (FP-ENAS), University Fernando Pessoa, Porto, Portugal

Correspondence: Ana Rita Lima (ritacmlima@hotmail.com) or Paula Guedes de Pinho (pguedes@ff.up.pt) or Márcia Carvalho (mcarv@ufp.edu.pt)
}

Received: 20 May 2019 Revised: 21 August 2019 Accepted: 4 September 2019

Published online: 7 October 2019 
profile may reflect modifications in gene activation, gene expression, proteins and activity of enzymes involved in metabolic pathways. These volatile molecules endow biological samples with distinct odours which may even be detected by animals with highly sensitive olfactory capabilities, such as dogs, ${ }^{11,12}$ or sophisticated analytical instrumental techniques, such as gas chromatography-mass spectrometry (GC-MS) combined with multivariate statistical analysis (MVA) ${ }^{8-10}$ In this regard, Smith et al. ${ }^{8}$ studied the urine metabolomics of 13 PCa patients and 24 controls using GC-MS, disclosing 91 VOCs and unveiling significant differences between PCa and controls in 21 VOCs. However, this study has important limitations namely a small sample size and lack of external validation. ${ }^{8}$ Khalid et al. performed the GC-MS volatile profiling of urine from PCa patients using a larger number of samples ( $n=59$ PCa and $n=43$ controls). Overall, 196 VOCs were identified from which four (2,6-dimethy-7-octen-2-ol, pentanal, 3-octanone, and 2-octanone) were found to be statistically different between PCa and control samples. ${ }^{9}$ More recently, Jimenez-Pacheco et al. performed a similar study using 29 PCa urine samples that were compared with 21 samples from patients with benign prostatic hyperplasia (BPH). In this study, 57 VOCs were identified, but only nine significantly differed between the two groups, highlighting furan and $\mathrm{p}$-xylene as potential PCa biomarkers. ${ }^{10}$ Interestingly, 2-octanone ${ }^{8,9}$ and 2,6-dimethy-7octen-2-ol $^{9,10}$ were pointed as urinary PCa biomarkers in more than one study. Taken together, these studies provide convincing evidence that volatiles emanating from urine are potential biomarkers for PCa detection. Recently, the feasibility and potential of volatile signature for diagnosing PCa led to the development of chemical system sensors (so-called "electronic nose" or "e-nose"). ${ }^{13,14}$ "E-noses" are designed to mimic the mammalian olfactory system and provide a global characterisation of the odorous mixtures. ${ }^{15}$ Remarkably, the application of the "enose" technology to discriminate the odour of urine from patients with PCa from controls provided better diagnostic performance than serum PSA. ${ }^{13,14}$

Herein, we aimed to obtain a more comprehensive metabolomic profiling of volatile metabolites in urine from PCa patients, using a metabolomics approach based on headspace solid-phase microextraction coupled with GC-MS (HS-SPME/GC-MS). Two different sample preparation strategies were considered: (i) direct analysis for VOCs detection and (ii) derivatisation with $0-(2,3,4,5,6-$ pentafluorobenzyl)hydroxylamine (PFBHA), prior to HS-SPME/GCMS analysis, to enhance the sensitive detection of volatile carbonyl compounds (VCCs). An external validation set was then used to validate a panel of discriminant volatile compounds with clinical potential for PCa diagnosis. To the best of our knowledge, this is the first time that VCCs are investigated as urinary PCa biomarkers and that a volatile biomarker panel for $\mathrm{PCa}$ is validated using an external set of samples.

\section{METHODS}

Chemicals

All chemicals used were of analytical grade. Benzaldehyde ( $\geq 99.5 \%)$, 2-butanone ( $\geq 99 \%)$, (E)-2-butenal ( $\geq 99 \%)$, cyclohexanone $(\geq 99 \%)$, 2-decanone ( $\geq 98 \%)$, (E)-2-decenal ( $\geq 92 \%), 2,5$-dimethylbenzaldhyde ( $\geq 99 \%)$, 3,4-dimethylcyclohex-3-ene-1-carbaldehyde ( $\geq 97 \%)$, 2,6-dimethyl-6-hepten-2-ol ( $\geq 96 \%)$, 3,7-dimethylocta-1,6dien-3-ol ( $\geq 95 \%)$, 4-fluorobenzaldehyde ( $\geq 98 \%)$, 2-furfural $(\geq 99 \%)$, heptanal $(\geq 92 \%)$, 4-heptanone $(\geq 97 \%)$, hexadecane $(\geq 99 \%),(E, E)$ 2,4 -hexadienal $(\geq 95 \%)$, hexanal $(\geq 98 \%)$, 2-hexanone $(\geq 98 \%)$, 2hydroxy-2-methyl-1-phenylpropan-1-one ( $\geq 97 \%)$, 2-methylbutanal $(\geq 90 \%)$, 3-methylbutanal $(\geq 97 \%), \quad$ 2-methylcyclopentan-1-one $(\geq 97 \%)$, 5-methyl-2-furfural ( $\geq 99 \%)$, methylglyoxal (40\% aqueous solution), 5-methylheptan-2-one ( $\geq 95 \%)$, 2-methylpropanal $(\geq 98 \%)$, 5-methyl-2-(propan-2-yl) cyclohexyl acetate ( $\geq 98 \%)$, nonanal $(\geq 95 \%)$, 2-nonanone ( $\geq 97 \%)$, (E)-2-nonenal $(\geq 93 \%)$, octanal
( $\geq 98 \%)$, 2-octanone ( $\geq 98 \%)$, pentanal $(\geq 97 \%)$, (E)-2-pentenal $(\geq 95 \%)$, 3-penten-2-one $(\geq 70 \%)$, 3-phenylpropionaldehyde $(\geq 95 \%)$, PFBHA $(\geq 98 \%)$, phenylacetaldehyde $(\geq 90 \%)$, propanal ( $\geq 97 \%$ ), terpinen-4-ol ( $\geq 95 \%), 2,6,6,10$-tetramethyl-1-oxaspiro[4.5] dec-9-ene ( $\geq 90 \%)$, and 3,7,7-trimethylbicyclo[4.1.0] hept-3-ene $(\geq 97 \%)$ were purchased from Sigma-Aldrich (Madrid, Spain). Butanal $(\geq 99 \%)$ and glyoxal $(\geq 95 \%)$ were purchased from Fluka (Madrid, Spain) and 4-hydroxy-2-nonenal ( $\geq 98 \%)$ was purchased from Cayman Chemical (USA). Sodium chloride was obtained from VWR (Leuven, Belgium).

\section{Subjects}

Early morning urine samples without fasting were collected from PCa patients and controls at the Portuguese Oncology Institute of Porto (IPO Porto) and frozen at $-80^{\circ} \mathrm{C}$ until analysis. The study protocol was approved by the local Ethics Committee and all subjects provided their signed informed consent prior to enrolment.

A cohort of 118 men were included in this study: $58 \mathrm{PCa}$ patients (age 52-77 years, mean 63) and 60 cancer-free control subjects (age 56-66 years, mean 59). Both PCa and control groups were randomly divided into two sets: (1) training ( $n=40$ PCa and $n=42$ controls for VOCs; $n=40$ PCa and $n=40$ controls for VCCs) and (2) external validation ( $n=18 \mathrm{PCa}$ and $n=18$ controls for VOCs and VCCs). Control group consisted of subjects with agerelated comorbidities such as hypertension, diabetes, lipid disorders and $\mathrm{BPH}$, but without cancer. Detailed information on Gleason score and some important biochemical and clinical parameters of $\mathrm{PCa}$ patients and control subjects is provided in Table 1.

Sample preparation and metabolites extraction

Urine samples were thawed at $4{ }^{\circ} \mathrm{C}$. For VOCs analysis, $1 \mathrm{~mL}$ of sample was placed in a $10 \mathrm{~mL}$ glass vial with $20 \mu \mathrm{L}$ of internal standard (IS) $(10 \mu \mathrm{g} / \mathrm{mL}$ 4-fluorobenzaldehyde in ultrapure water) and $\mathrm{NaCl}(0.27 \mathrm{~g})$. To optimise the extraction conditions, a central composite design (CCD) was performed (data not shown). The optimal extraction conditions, using divinylbenzene/carboxen/ polydimethylsiloxane (DVB/CAR/ PDMS) fiber coating, were $11 \mathrm{~min}$ of incubation and $30 \mathrm{~min}$ of extraction at $44^{\circ} \mathrm{C}$ under continuous stirring (250 rpm).

For VCCs analysis, $250 \mu \mathrm{L}$ of urine were placed in a $10 \mathrm{~mL}$ glass vial with $5 \mu \mathrm{L}$ of IS $(10 \mu \mathrm{g} / \mathrm{mL}$ 4-fluorobenzaldehyde in ultrapure water) and $7.5 \mu \mathrm{L}$ of the derivatizing agent PFBHA $(40 \mathrm{~g} / \mathrm{L}$ in ultrapure water). Extraction was performed according to the conditions previously optimised in our lab ${ }^{16}$ using a CombiPAL automatic autosampler (Varian, Palo Alto, CA) and a polydimethylsiloxane/divinylbenzene (PDMS/DVB) fiber coating. Briefly, urine samples were incubated at $62^{\circ} \mathrm{C}$ during $6 \mathrm{~min}$, followed by extraction of volatiles at the same temperature during $51 \mathrm{~min}$, under continuous stirring ( $250 \mathrm{rpm})$. After extraction, the fiber was inserted into the GC system for thermal desorption of the analytes at $250{ }^{\circ} \mathrm{C}$ during $5 \mathrm{~min}$.

In both approaches, all samples were randomly injected, with the quality control (QCs) samples being injected at the same conditions on every eight samples. QCs were prepared as aliquots of a pool of all urine samples (PCa and controls) considered in this study.

\section{GC-MS analysis}

A Scion 436-gas chromatograph coupled to a Bruker single quadrupole (SQ) equipped with a Scion SQ ion trap mass detector and a Bruker Daltonics MS workstation software version 6.8 , with a Rxi-5Sil MS (30 $\mathrm{m} \times 0.25 \mathrm{~mm} \times 0.25 \mu \mathrm{m})$ column from RESTEK were used. Briefly, the carrier gas was helium C-60 (Gasin, Portugal) (flow rate $1 \mathrm{~mL} / \mathrm{min}$ ) and the injector port was heated at $230^{\circ} \mathrm{C}$. The oven temperature was fixed at $40^{\circ} \mathrm{C}$ for $1 \mathrm{~min}$, increasing to $250^{\circ} \mathrm{C}$ (rate $5^{\circ} \mathrm{C} / \mathrm{min}$ ), held for $5 \mathrm{~min}$, followed by increasing to 


\begin{tabular}{|c|c|c|c|c|c|c|c|c|}
\hline \multirow[t]{2}{*}{ Characteristics } & \multicolumn{4}{|c|}{ Prostate cancer } & \multicolumn{4}{|l|}{ Control } \\
\hline & $\begin{array}{l}\text { Training } \\
\text { set VOCs }\end{array}$ & $\begin{array}{l}\text { External } \\
\text { set VOCs }\end{array}$ & $\begin{array}{l}\text { Training } \\
\text { set VCCs }\end{array}$ & $\begin{array}{l}\text { External } \\
\text { set VCCs }\end{array}$ & $\begin{array}{l}\text { Training } \\
\text { set VOCs }\end{array}$ & $\begin{array}{l}\text { External } \\
\text { set VOCs }\end{array}$ & $\begin{array}{l}\text { Training set } \\
\text { VCCs }\end{array}$ & $\begin{array}{l}\text { External set } \\
\text { VCCs }\end{array}$ \\
\hline Number of subjects & 40 & 18 & 40 & 18 & 42 & 18 & 40 & 18 \\
\hline $\begin{array}{l}\text { Mean Age } \pm \text { SD } \\
\text { (years) }\end{array}$ & $64.4 \pm 6.4$ & $61.8 \pm 5.2$ & $63.7 \pm 6.5$ & $63.4 \pm 5.3$ & $59.3 \pm 3.0$ & $59.6 \pm 2.62$ & $59.3 \pm 2.8$ & $59.8 \pm 2.7$ \\
\hline$<4$ & $3(7.5 \%)$ & $1(5.6 \%)$ & - & $4(22.2 \%)$ & - & - & - & - \\
\hline $4-10$ & $24(60 \%)$ & $13(72.2 \%)$ & $28(70 \%)$ & $9(50 \%)$ & - & - & - & - \\
\hline$>10$ & $13(32.5 \%)$ & $4(22.2 \%)$ & $12(30 \%)$ & $5(27.8 \%)$ & - & - & - & - \\
\hline \multicolumn{9}{|l|}{ Gleason score, $n$ (\%) } \\
\hline$\leq 6$ & $6(15 \%)$ & $3(16.7 \%)$ & $8(20 \%)$ & $1(5.6 \%)$ & - & - & - & - \\
\hline II & - & $2(11.1 \%)$ & $2(4 \%)$ & - & - & - & - & - \\
\hline$\| A$ & 7 (17.5\%) & $4(22.2 \%)$ & $9(22.5 \%)$ & $2(11.1 \%)$ & - & - & - & - \\
\hline IIB & 15 (37.5\%) & $2(11.1 \%)$ & $11(27.5 \%)$ & $6(33.3 \%)$ & - & - & - & - \\
\hline III & $13(32.5 \%)$ & $5(27.8 \%)$ & $10(25 \%)$ & $8(44.4 \%)$ & - & - & - & - \\
\hline IV & $2(5 \%)$ & $2(11.1 \%)$ & $4(10 \%)$ & - & - & - & - & - \\
\hline Alcoholism, $n$ (\%) & 7 (17.5\%) & $4(22.2 \%)$ & $9(22.5 \%)$ & $2(11.1 \%)$ & $3(7.1 \%)$ & - & $2(5 \%)$ & $1(5.6 \%)$ \\
\hline Smoking, $n$ (\%) & $2(5 \%)$ & - & $2(5 \%)$ & - & $5(11.9 \%)$ & $2(11.1 \%)$ & $6(15 \%)$ & $1(5.6 \%)$ \\
\hline Obesity, n (\%) & $6(15 \%)$ & $4(22.2 \%)$ & 7 (17.5\%) & $3(16.7 \%)$ & 7 (16.7\%) & $3(16.7 \%)$ & 7 (17.5\%) & $2(11.1 \%)$ \\
\hline $\begin{array}{l}\text { Cardiac condition, } \\
n(\%)\end{array}$ & $5(12.5 \%)$ & $6(33.3 \%)$ & 7 (17.5\%) & $4(22.2 \%)$ & - & 1 (5.6\%) & - & $1(5.6 \%)$ \\
\hline
\end{tabular}

$300^{\circ} \mathrm{C}$ (rate $5^{\circ} \mathrm{C} / \mathrm{min}$ ) and held for $1 \mathrm{~min}$. The temperatures of transfer line, manifold and trap were $280^{\circ} \mathrm{C}, 50^{\circ} \mathrm{C}$ and $180^{\circ} \mathrm{C}$, respectively. The emission current was $50 \mu \mathrm{A}$ and the electron multiplier was set in relative mode to an auto tune procedure. All mass spectra were acquired in the electron impact mode $\left(270^{\circ} \mathrm{C}\right)$. The analysis was performed in full scan mode and the mass range used was $40-350 \mathrm{~m} / \mathrm{z}$, with a scan rate of $6 \mathrm{scan} / \mathrm{s}^{17}$

To analyse VCCs, a 436-GC model (Bruker Daltonics) coupled to an EVOQ triple quadrupole mass spectrometer (Bruker Daltonics) and a Bruker MS workstation software version 8.2 were used. The chromatographic separation was accomplished using a fused silica capillary column (Rxi-5Sil MS; $30 \mathrm{~m} \times 0.25 \mathrm{~mm} \times 0.25 \mu \mathrm{m}$; Restek Corporation, U.S., Bellefonte, Pennsylvania) and high purity helium C-60 (Gasin, Portugal) as carrier gas (flow rate $1 \mathrm{~mL} / \mathrm{min}$ ). The oven temperature was held at $40^{\circ} \mathrm{C}$ for $1 \mathrm{~min}$, increasing to $250^{\circ} \mathrm{C}$ (rate $5^{\circ} \mathrm{C} / \mathrm{min}$ ), held for $5 \mathrm{~min}$, finally increasing to $300^{\circ} \mathrm{C}$ (rate $20^{\circ} \mathrm{C}$ / min). The temperature of transfer line and manifold were $260^{\circ} \mathrm{C}$ and $40^{\circ} \mathrm{C}$, respectively. The emission current was $50 \mu \mathrm{A}$ and the electron multiplier was set in relative mode to an auto tune procedure. All mass spectra were acquired in the electron impact mode $\left(270{ }^{\circ} \mathrm{C}\right)$. Data acquisition was performed in full scan mode and a $50-600 \mathrm{~m} / \mathrm{z}$ mass range was used. ${ }^{16}$
The metabolite identification was accomplished by comparison of the MS spectra with standards (whenever available), the National Institute of Standards and Technology (NIST 14) database spectral library, and comparison of the experimental and theory (literature) Kovats index.

Data pre-processing

Before statistical analysis, the data was pre-processed using MZmine $2,^{18}$ including baseline correction, peak detection, chromatogram deconvolution and alignment. The parameters used for pre-processing of VOCs data were: RT range $2.0-29.0 \mathrm{~min}$, $\mathrm{m} / \mathrm{z}$ range $50-400, \mathrm{MS}$ data noise level $1.0 \times 10^{5}, \mathrm{~m} / \mathrm{z}$ tolerance 0.2 , chromatogram baseline level $1.0 \times 10^{2}$ and peak duration range 0.06-0.70 min; whereas for VCCs were: RT range $6.5-38.0 \mathrm{~min}, \mathrm{~m} / \mathrm{z}$ range $50-600$, MS data noise level $5.0 \times 10^{5}, \mathrm{~m} / \mathrm{z}$ tolerance 0.2 , chromatogram baseline level $1.0 \times 10^{4}$ and peak duration range $0.06-0.70 \mathrm{~min}$. In both approaches, all RT $-\mathrm{m} / \mathrm{z}$ pairs with a relative standard deviation greater than $30 \%$ in QCs, as well as RT-m/z pairs identified as contaminants (from column, fiber, among others), were manually removed from the matrix. The obtained data were normalised by the total area of the chromatograms and the final matrix was scaled to pareto. Furthermore, to reduce the 
variation from uncontrolled confounding factors and simplify the data, a variable selection method based in a univariate test, ${ }^{19}$ namely $t$-test, was performed using MetaboAnalyst. ${ }^{20}$ Consequently, all variables with $p$-value $>0.05$ were removed from the matrix.

\section{Statistical analysis}

The statistical analysis strategy used for VOCs and VCCs data was similar and included multivariate and univariate statistical tests. From all available samples, $70 \%$ were used for the training set and $30 \%$ were randomly selected for the external set. MVA was performed using the training set and included principal component analysis (PCA) and partial least squares discriminant analysis (PLS-DA) in SIMCA-P 15 (Umetrics, Sweden). The robustness of the PLS-DA models was confirmed through 7-fold cross validation and permutation test (200 random permutations of Y-observations, 2 components) (SIMCA-P 15, Umetrics, Sweden). To test the validity of the created models, an internal (training set) and external (external set) validation was performed. For internal and external validations, receiver operating characteristic curves (ROC), area under the curve (AUC), sensitivity, and specificity were computed (MetaboAnalyst) $^{20}$ for both PLS-DA models (VOCs and VCCs). The samples of the external set were classified as cancer or controls, taking into consideration the PLS-DA models obtained using the training sets and the sensitivity, specificity and accuracy of both PLS-DA models (VOCs and VCCs) were computed. ${ }^{21}$

After MVA, all metabolites with VIP (Variable Importance to the Projection) greater than one were subjected to univariate analysis (GraphPad Prism 6, USA), including a normality test (Shapiro-Wilk test) followed by unpaired Student's $t$-test with Welch correction test, for normal distribution, or unpaired Mann-Whitney U-test, for non-normal distribution. Percentage of variation, uncertainty of the percentage of variation, and effect size and the standard error were also determined. ${ }^{22}$ For all significantly altered metabolites ( $p$ value $<0.05$ and effect size higher than the standard error), receiver operating characteristic curves $(\mathrm{ROC})$, area under the curve (AUC), sensitivity, and specificity were also computed (MetaboAnalyst). ${ }^{20}$ Bonferroni correction was used to adjust $p$ values in multiple comparisons. ${ }^{23}$ Multivariate ROC exploratory analysis (Metaboanalyst) ${ }^{20}$ was used to define a small panel of discriminant metabolites with high accuracy for prostate cancer detection, envisaging a possible translation into clinics using an "e-nose". The PLS-DA algorithm was used to evaluate the importance of each discriminant metabolite based on VIP scores through repeated random sub-sampling cross validation. The top important metabolites were used to build a PLS-DA model which was validated through ROC analysis using the training and external sets.

To better understand the biological relevance of the significantly altered VOCs and VCCs, a metabolic pathway analysis using the MetPa tool was performed in Metaboanalyst. ${ }^{20}$ Finally, to search for possible correlations between the metabolites significantly altered in PCa, Spearman's rank correlation coefficient was computed for the set of identified and putatively annotated statistically significant compounds and represented in a heatmap, using $\mathrm{R}$ software (version 3.5.1). ${ }^{24}$ Spearman's rank correlation coefficient was also computed between age and the set of metabolites found altered in PCa compared to controls.

\section{RESULTS}

Urinary volatile profile of PCa patients vs. controls In this study, a HS-SPME/GC-MS method was employed to evaluate differences in the urinary volatile profile of PCa patients compared with controls. To accomplish a more comprehensive evaluation of the urinary volatilome, we used two different sample preparation techniques which enabled the identification of 122 VOCs and 148 VCCs (seven common compounds were found).
MVA was used to evaluate the reproducibility of both analytical strategies and the discriminant capability of the PLS-DA models created using the training set. The QC samples were closely clustered in the PCA scores scatter plot (Fig. S1), which confirmed the analytical reproducibility of both methods. For construction of the PLS-DA models, a variable selection method was performed (VOCs: 3232 variables x 82 samples; VCCs: 246 variables $x$ 80 samples) to improve the prediction power. In Fig. 1, the discriminant capability of the PLS-DA models, after variable selection, is clearly observed (VOCs model: $L V=2 ; R^{2} X=0.172$; $\mathrm{R}^{2} \mathrm{Y}=0.776 ; \mathrm{Q}^{2}=0.599 ; \mathrm{VCC}$ model: $\mathrm{LV}=2 ; \mathrm{R}^{2} \mathrm{X}=0.354 ; \mathrm{R}^{2} \mathrm{Y}=$ $\left.0.534 ; \mathrm{Q}^{2}=0.443\right)$. Model robustness was also confirmed through permutation testing (Fig. S2). In the internal validation, VOCs PLSDA model showed an AUC of 0.975 , a sensitivity of $92 \%$ and specificity of $100 \%$ and the VCCs model unveiled an AUC of 0.878 , a sensitivity of $71 \%$ and specificity of $91 \%$ (Fig. 1).

Furthermore, an external validation set was used to confirm the validity of the training models. For VOCs and VCCs, among $18 \mathrm{PCa}$ samples, 14 were accurately classified and four were poorly classified. On the other hand, 17 control samples were accurately classified and only one was poorly classified for VOCs, whereas all 18 control samples were correctly classified for VCCs (Table S3). Thus, taking into consideration these results, a sensitivity of $78 \%$, a specificity of $94 \%$ and an accuracy of $86 \%$ was obtained for VOCs, whereas VCCs disclosed equal sensitivity, a specificity of $100 \%$ and an accuracy of $89 \%$. For VOCs, from a total of 64 metabolites with VIP $>1,31$ were found significantly different between the two groups (PCa vs. control). The discriminant VOCs included three aldehydes, six ketones, two alcohols, two monoterpene alcohols, one alkene, one cycloalkane, two terpenes, among others, and 11 unidentified compounds (Table 2). Regarding VCCs analysis, 21 metabolites showed VIP $>1$ and 12 significantly differed between PCa and control groups. The discriminant VCCs included two alpha-ketoaldehydes, one alkanal, one alkenal, two aromatic aldehydes, three ketones, one alkane and two unidentified compounds (Table 3). The chromatographic characteristics considered for identification of VOCs and VCCs are displayed in Tables S1 and S2, respectively. AUC values were superior to 0.6 for all statistically significantly altered metabolites (Tables 2 and 3). The sensitivity and specificity of the individual metabolites was also determined and, despite the lower individual sensitivity and specificity found for the majority of the metabolites when compared to the one obtained for the models (Fig. 1 and Table S3), all metabolites disclosed sensitivity and specificity greater than 50 and $70 \%$, respectively (Tables 2 and 3 ).

Age (Table 1) significantly differed between PCa and controls in VOCs (Mann-Whitney test $p$-value $=0.0002$ ) and VCCs (Mann-Whitney test $p$-value $=0.0022$ ) training sets. Hence, a possible influence of age in the set of metabolites found altered in PCa compared to controls (Tables 2 and 3) was investigated through Spearman correlation, unveiling no statistically relevant correlations $(|r| \leq 0.36)$ (Table S4). In addition, the number of individuals with arterial hypertension $(\mathrm{AH})$ was higher in $\mathrm{PCa}$ group compared to controls in the VOCs training set (Table 1). The impact of $\mathrm{AH}$ on urine volatile profile was evaluated in the control group ( $\mathrm{AH} n=14$ vs. non-HA $n=28$ ), revealing no predictive power $\left(Q^{2}=-0.145\right)$ in the PLS-DA model (Fig. S3). Taking into consideration these results, no age- and $\mathrm{AH}$-related changes were found in the urinary volatile signature of PCa patients.

Definition of a multi-biomarker panel for PCa diagnosis The smallest panel of metabolites that best predict PCa comprised 6 metabolites, namely hexanal, 2,5-dimethylbenzaldehyde, 4methylhexan-3-one, dihydroedulan IA, methylglyoxal and 3phenylpropionaldehyde. This panel showed an AUC of 0.856 , a sensitivity of $72 \%$, a specificity of $96 \%$ and an accuracy of $79 \%$ taking into consideration the internal validation (Fig. 2). Regarding the external validation set, the 6-biomarker panel showed an AUC 


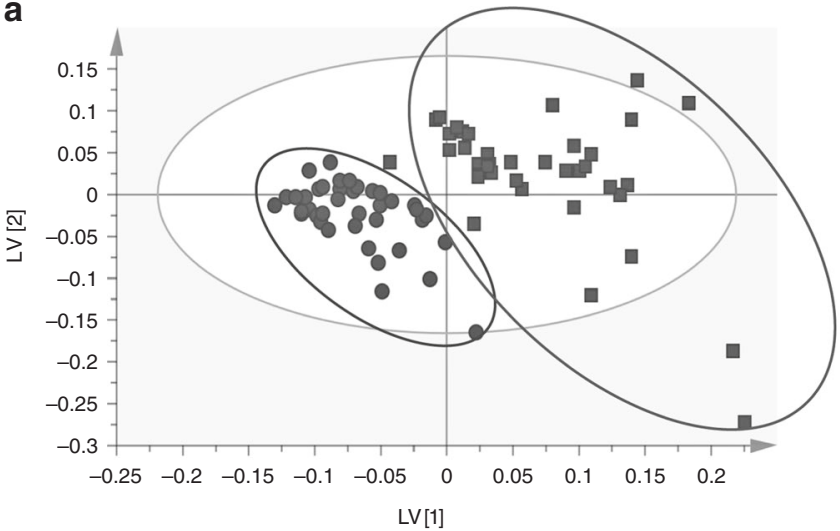

$\mathrm{LV}=2, R^{2} X=0.172 ; R^{2} Y=0.776 ; Q^{2}=0.599$

C

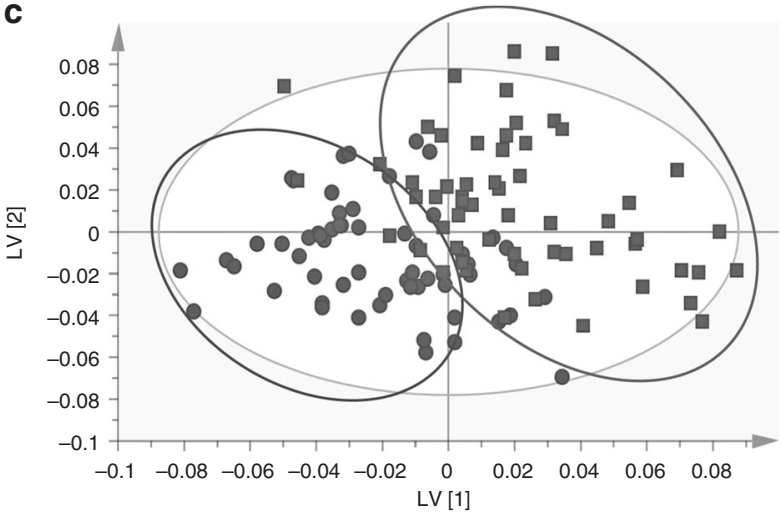

$\mathrm{LV}=2, R^{2} X=0.354 ; R^{2} Y=0.534 ; Q^{2}=0.443$ b

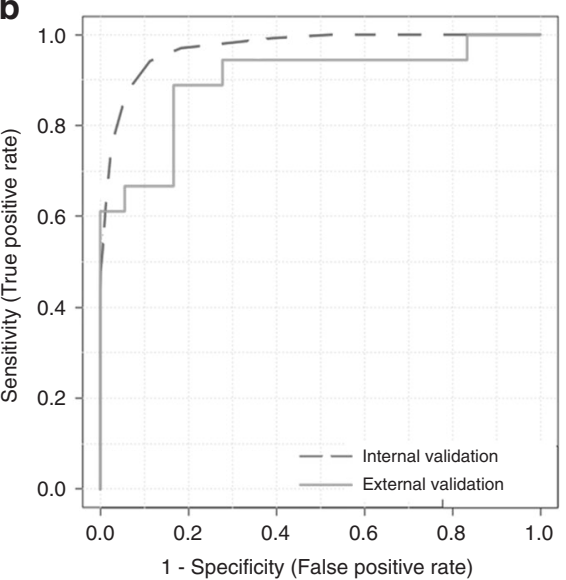

d

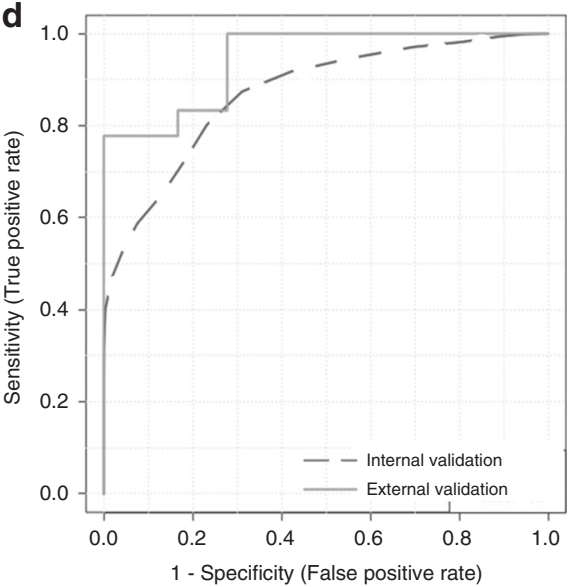

Fig. 1 a PLS-DA scores scatter plot (Pareto scaling; 2 components) obtained for VOCs training model of PCa patients ( $n=40$, squares) vs. cancer-free controls ( $n=42$, circles), after variable selection; $\mathbf{b}$ Assessment of the diagnostic performance of the PLS-DA model obtained for VOCs using the training set (AUC $=0.975$; sensitivity $=92 \%$; specificity $=100 \%$ ) and the external set $(A U C=0.898$; sensitivity $=78 \%$; specificity $=94 \%$ ) through ROC analysis; c PLS-DA scores scatter plot (Pareto scaling; 2 components) obtained for VCCs training model of PCa patients ( $n=40$, squares) vs. cancer-free controls ( $n=40$, circles), after variable selection; $\mathbf{d}$ Assessment of the diagnostic performance of the PLS-DA model obtained for VCCs using the training set (AUC $=0.878$; sensitivity $=71 \%$; specificity $=97 \%)$ and the external set $(A U C=0.944$; sensitivity $=78 \%$; specificity $=100 \%$ ) through ROC analysis

of 0.904 , a sensitivity of $89 \%$, a specificity of $83 \%$ and an accuracy of $86 \%$ (Fig. 2 and Table S5).

Although integration of volatile compounds in specific biochemical pathways is still difficult to accomplish, MetPA tool ${ }^{20}$ was used for identification of the most relevant metabolic pathways where the discriminant compounds are involved. The results revealed that methylglyoxal is involved in pyruvate metabolism and glycine, serine and threonine metabolism, phenylacetaldehyde in phenylalanine metabolism and hexanal in steroid hormone biosynthesis (Fig. S4).

To overcome the lack of knowledge about the role of volatile compounds in the metabolic pathways, Spearman's correlation indexes were computed using all identified metabolites (L1 and L2 in Tables 2, 3, S1 and S2) significantly altered in urine of PCa patients (Fig. 3). The magnitude and the sign of correlations can provide identification of metabolites in the same metabolic pathway or under some common regulatory mechanisms. Stronger positive correlations $(r>0.7$ and $p<0.0001)$ were observed for 2,6,6,10-tetramethyl-1-oxaspiro[4.5]dec-9-ene with 5-methyl-2-(propan-2-yl)cyclohexyl acetate $(r=0.75)$, hexadecane with cyclohexanone $(r=0.72)$, 3-phenylpropionaldehyde with cyclohexanone $(r=0.77)$, 3-phenylpropionaldehyde with hexadecane $(r=0.71)$ and 3-phenylpropionaldehyde with phenylacetaldehyde $(r=0.76)$.

\section{DISCUSSION}

In this study, two HS-SPME/GC-MS approaches were used to more comprehensively uncover the volatile profile of urine from $\mathrm{PCa}$ patients compared with previous reports, ${ }^{8-10}$ unveiling a total of 263 different volatile compounds. Multivariate analysis showed that both VOCs and VCCs urinary signature allowed for accurate discrimination between PCa and control groups. A major strength of this study lies in its design, with the inclusion of an external validation set to validate the models obtained through MVA of the training sets, after variable selection. These external validation sets disclosed satisfactory sensitivity (78\% for VOCs and VCCs), high specificity (94\% for VOCs and $100 \%$ for VCCs) and high accuracy (86\% for VOCs and $89 \%$ for VCCs). Interestingly, all false negatives observed in VOCs model were from obese and/or alcoholic subjects, whereas the false positive was a control with prostatitis (Table 1). Among the four false negatives observed in VCCs model, three were also obese subjects and one with ischaemic heart disease, which may compromise renal function (Table 1). These confounding factors might justify the misclassifications. Notwithstanding, specificity and accuracy were superior to previously published in similar studies. ${ }^{8,9}$ Furthermore, individually, all discriminant metabolites disclosed sensitivity (ranging from 48 to $80 \%$; Tables 2 and 3 ) higher than the one reported for serum PSA (20.5\%). ${ }^{4}$ 


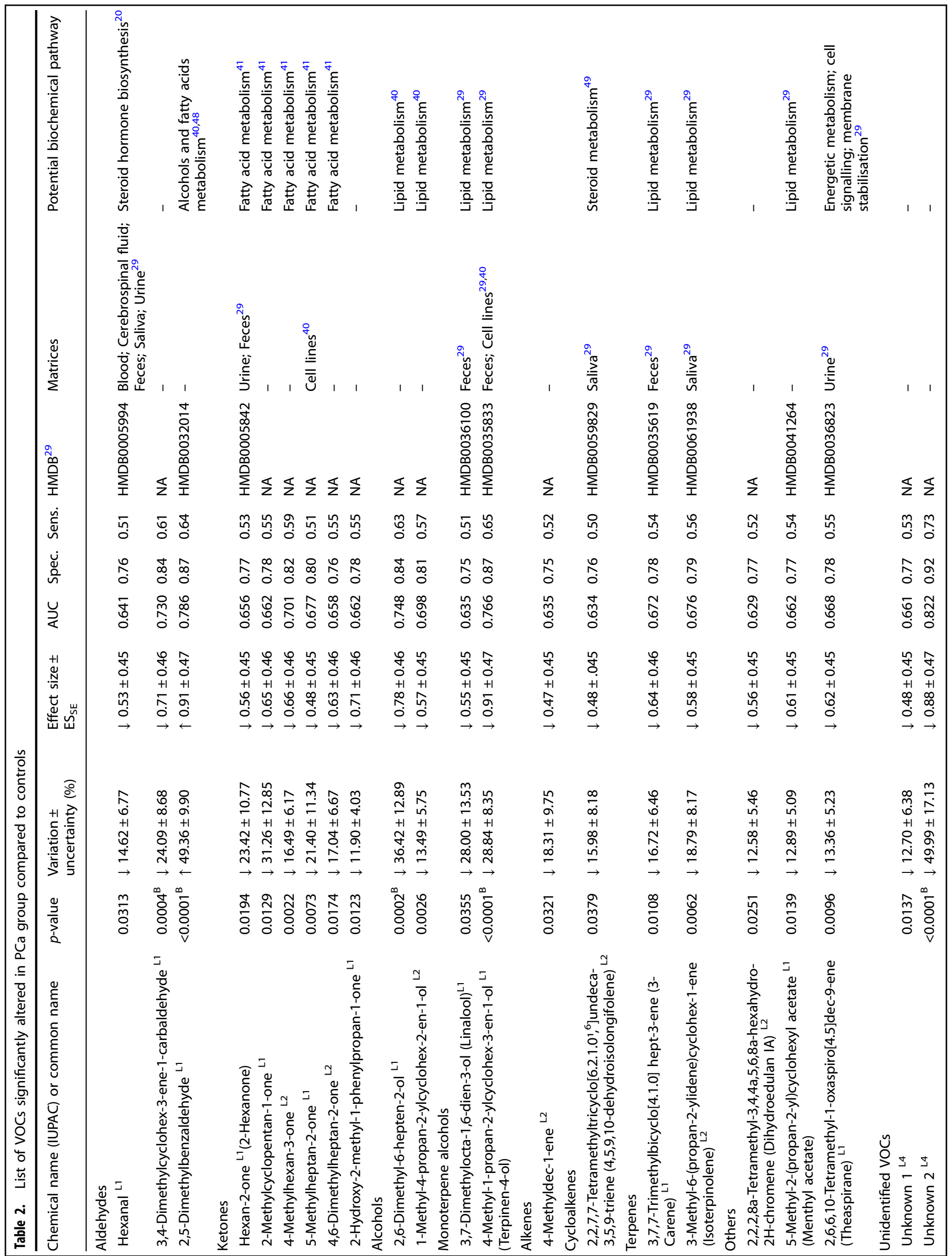


The idea of using multiple biomarkers rather than a single biomarker has gained strength as a means to improved performance, ${ }^{25}$ since the metabolomic signature of a disease is comprised of groups of connected metabolites that change in concert. ${ }^{26}$ Furthermore, this approach ensures that an arbitrary change in a single metabolite will not lead to a false diagnosis. ${ }^{26}$ In line with this, a biomarker panel was herein defined consisting in the combination of 6 discriminatory metabolites. A small panel of biomarkers was selected in this work envisaging the development of a sensing material ${ }^{27}$ tuned in specificity and selectivity for these compounds to be applied in an "e-nose" in near future. This 6biomarker panel unveiled good prediction of PCa from non-cancer patients, providing accuracies of $79 \%$ and $86 \%$ in the internal and external sets, respectively. The small sample size in external set can be considered a limiting factor in this study, though this is the first study, to our knowledge, to use an external set for validation of a volatile biomarker panel of PCa in urine. Importantly, the four patients with $\mathrm{BPH}$ and one patient with prostatitis included in the external set as controls were correctly classified by the panel. These prostate non-malignant conditions are well-recognised confounders in the context of serum PSA screening, as elevated levels of this biomarker are detected in BPH and prostatitis. ${ }^{25}$ So, taking into consideration the results of the internal and external validations, the diagnostic performance of the 6-biomarker panel outperforms not only PSA sensitivity but also fPSA/tPSA sensitivity and specificity.

In our study, three classes of compounds stood out as discriminant of PCa from controls, namely alcohols, aldehydes and ketones. A significant decrease was found in the levels of four alcohols, specifically terpinen-4-ol, 2,6-dimethyl-6-hepten-2-ol, 1methyl-4-propan-2-ylcyclohex-2-en-1-ol, and 3,7-dimethylocta-1,6dien-3-ol (Table 2). This may be related with changes in several metabolic pathways, namely hydrocarbon metabolism, ${ }^{28}$ fatty acid $\beta$-oxidation, ${ }^{29}$ intensification of cellular membrane synthesis ${ }^{30}$ and alterations in the activity of some important enzymes, namely CYP $450^{31}$ and alcohol dehydrogenases. ${ }^{28}$ Several studies have demonstrated the intracellularly increased concentrations of reactive oxygen species (ROS) in cancer cells, ${ }^{32,33}$ which are capable of causing the oxidation of biologically crucial molecules such as DNA, RNA, proteins and lipids. ROS-mediated oxidation of polyunsaturated fatty acids (also termed lipid peroxidation) increases alkanes formation, which after hydroxylation through CYP 450 leads to the production of alcohols. ${ }^{31}$ Additionally, it has been proposed that terpinen-4-ol and a-terpineol (an isomer of terpinen-4-ol) can interfere with immune response, as they were able to inhibit the production of inflammatory mediators. ${ }^{34}$ Furthermore, a-terpineol was shown to have cytotoxic and apoptotic effects in PCa cell lines, which may be correlated with down-regulation of various proteins that mediate cell proliferation, cell survival, metastasis, and angiogenesis. ${ }^{35}$ 3,7-Dimethylocta-1,6-dien-3-ol may have an exogenous source, since it is present in several food products like cinnamon or citrus fruits. ${ }^{29}$ However, an endogenous origin cannot be ruled out since this compound is involved in lipid metabolism. ${ }^{29}$ In addition, the supplementation with 3,7-dimethylocta-1,6-dien-3-ol in PCa immortalised cell lines and in tumour xenografts showed an induction of apoptosis and inhibition of cell proliferation. ${ }^{36}$

Referring to aldehydes, urinary levels of hexanal, 3,4-dimethylcyclohex-3-ene-1-carbaldehyde, glyoxal, methylglyoxal, decanal, and 2-butenal were found significantly decreased in PCa patients, whereas 2,5-dimethylbenzaldehyde, 3-phenylpropionaldehyde and phenylacetaldehyde were significantly increased in $\mathrm{PCa}$ compared to controls (Tables 2 and 3). Aldehydes are involved in the metabolism of alcohols and fatty acids, ${ }^{37,38}$ and can also be produced during amino acid and carbohydrate catabolism. ${ }^{37,38}$ The presence of aldehydes may also be related with the excessive production of ROS, ${ }^{9}$ known to induce lipid peroxidation, which originates the formation of over 200 types of highly reactive and 


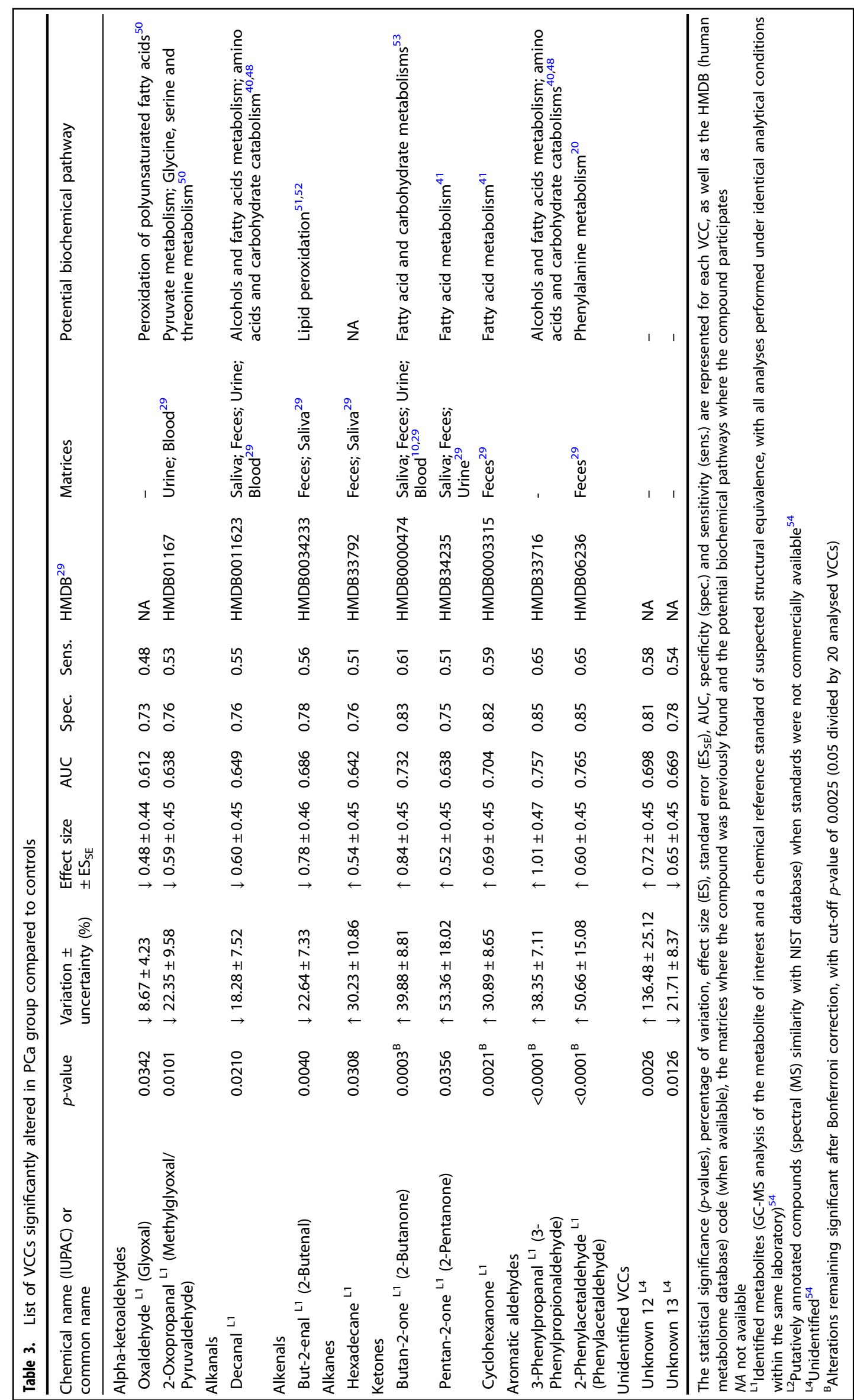




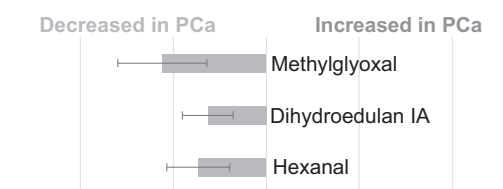

3-Phenylpropionaldehyde $\longrightarrow$

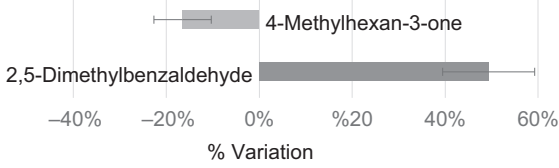

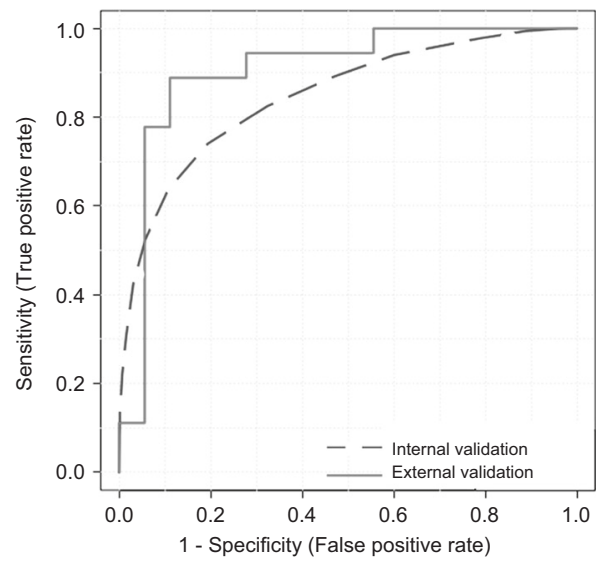

Fig. 2 Description, \% of variation and assessment of the diagnostic performance of the 6-biomarker panel using the training (AUC $=0.856$; sensitivity $=72 \%$; specificity $=96 \%$ ) and the external $(A U C=0.904$; sensitivity $=89 \%$; specificity $=83 \%$ ) sets through ROC analysis

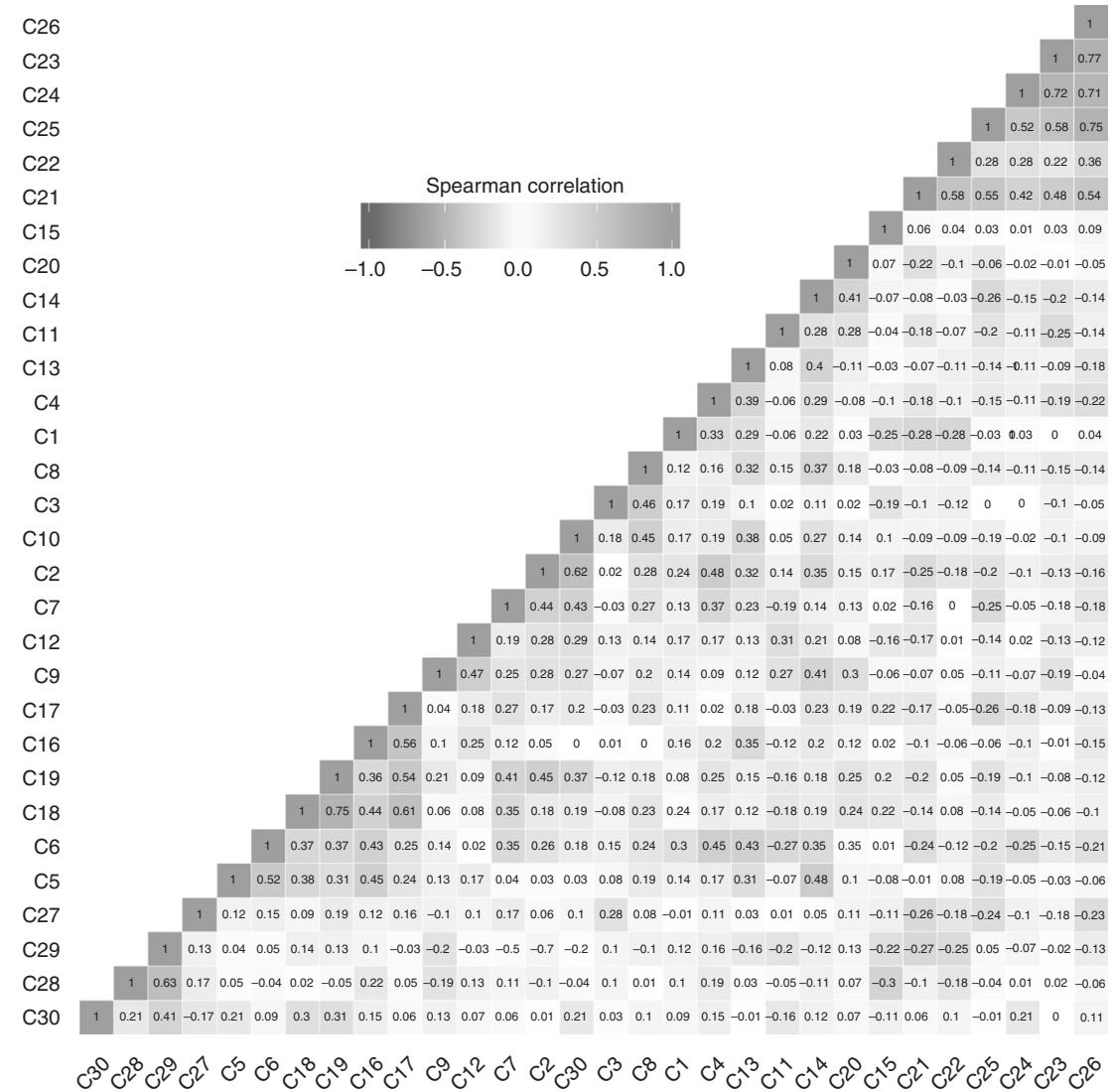

Fig. 3 Heatmap with the Spearman's correlations among the 30 identified and putatively identified metabolites significantly altered. C1: 2hexanone; C2: hexanal; C3: 2-methylcyclopentan-1-one; C4: 4-methylhexan-3-one; C5: 5-methylheptan-2-one; C6: 4-methyldec-1-ene; C7: 3,7,7-trimethylbicyclo[4.1.0] hept-3-ene; C8: 2,6-dimethyl-6-hepten-2-ol; C9: 3-methyl-6-(propan-2-ylidene)cyclohex-1-ene; C10: 4,6-dimethylheptan-2-one; C11: 3,7-dimethylocta-1,6-dien-3-ol; C12: 3,4-dimethylcyclohex-3-ene-1-carbaldehyde; C13: 1-methyl-4-propan-2-ylcyclohex-2en-1-ol; C14: terpinen-4-ol; C15: 2,5-dimethylbenzaldehyde; C16: 2-hydroxy-2-methyl-1-phenylpropan-1-one; C17: dihydroedulan IA; C18: 5-methyl-2-(propan-2-yl)cyclohexyl acetate; C19: 2,6,6,10-tetramethyl-1-oxaspiro[4.5]dec-9-ene; C20: 4,5,910-dehydroisolongifolene; C21: 2butanone; C22: 2-pentanone; C23: cyclohexanone; C24: hexadecane; C25: phenylacetaldehyde; C26: 3-phenylpropionaldehyde; C27: 2butenal; C28: decanal; C29: glyoxal; C30: methylglyoxal

extremely toxic aldehydes. ${ }^{39}$ This may explain the higher levels of 2,5-dimethylbenzaldehyde, 3-phenylpropionaldehyde and phenylacetaldehyde detected in urine of PCa patients. In agreement with our findings, other metabolomic studies have also observed a trend for increased production of certain aldehydes in $\mathrm{PCa}$ compared to control groups. ${ }^{8-10}$
The levels of nine ketones were also found significantly altered in urine from PCa patients, including 2-hexanone, 2-methylcyclopentan-1-one, 4-methylhexan-3-one, 5-methylheptan-2-one, 4,6-dimethylheptan-2-one, 2-hydroxy-2-methyl-1-phenyl-propan1-one, 2-butanone, 2-pentanone and cyclohexanone (Tables 2 and 3). Of note, increased levels of 2 -butanone ${ }^{10}$ and decreased 
5-methylheptan-2-one levels ${ }^{40}$ were previously associated with PCa in urine samples and cell lines, respectively. Alterations in the levels of ketones might be related with carcinogenic processes, such as protein metabolism and ketogenic pathway dysregulations. ${ }^{28}$ Some important ketones present in the human body are products of fatty acid metabolism, having acetyl-CoA as a precursor. ${ }^{41}$ The increase in ketone levels can also be associated with high oxidation rate of fatty acids and glycation. ${ }^{42}$ During glycation, ROS are formed and contribute to the glycation-induced protein modifications, normally designated glycoxidation. ${ }^{43}$

The exact metabolic pathways which constitute the biological origin of VOCs and VCCs is not completely elucidated yet. Thus far, only one study reported on the cancer-specific biochemical origin of VOCs. ${ }^{44}$ This goal is very difficult to accomplish as VOCs are produced during metabolic cascades as degradation products of the metabolites directly involved in metabolic pathways, and, consequently, conservative methods are unable to determine the VOCs real metabolic origin. ${ }^{44}$ Notwithstanding, some metabolites altered in the PCa group were associated with known biochemical pathways, namely pyruvate metabolism, glycine, serine and threonine metabolism, phenylalanine metabolism and steroid hormone biosynthesis (Fig. S4). However, it is important to take into account that some of the significantly altered metabolites may not be directly cancer-derived but reflect other local or systemic body responses (e.g., inflammation and/or necrosis).

Considering the correlation coefficient (Fig. 3) observed among all identified metabolites (L1 and L2 in Tables 2 and 3 and Tables S1 and S2) found significantly different between cancer and control, the significant decrease in the levels of $2,6,6,10$ tetramethyl-1-oxaspiro[4.5]dec-9-ene correlated with the significant decrease in the levels of 5-methyl-2-(propan-2-yl)cyclohexyl acetate, suggesting a possible relationship in PCa disturbed biochemical pathways. Furthermore, we also observed several strong correlations between alterations found in the levels of ketones, aldehydes and alkanes, suggesting a probable association of these compounds with PCa altered metabolism.

Despite the small sample size that may lead to bias in statistical power and precision, our results disclose a volatile biomarker panel that has the potential to be used as a non-invasive diagnostic tool for PCa with good performance. Notwithstanding, the use of a GC-MS approach in routine clinical practice has important limitations, including high cost, non-portability, timeconsuming process, and the need for considerable operator expertise. ${ }^{45}$ To overcome these limitations, the use of portable gas-sensing devices such as "e-noses" is a more suitable approach for routine clinical use. ${ }^{45}$ Some research groups have already demonstrated that "e-nose" technology is able to detect the "odour fingerprint" emanated from urine of PCa patients in a simple and fast way. ${ }^{13,14}$

The knowledge on the urinary volatile signature of PCa acquired with this study has the potential to allow for the development of a sensor optimised for the recognition of volatiles with chemical groups herein elucidated and consequently with greater capabilities of chemical discriminations and diagnostic accuracy. However, enose devices are incapable to determine the identity and concentration of individual compounds responsible for discrimination between urine samples and, therefore, do not provide information about the metabolic pathways affected by the disease. ${ }^{46}$ Furthermore, the reproducibility of "e-nose" results can be affected by sensor drift over time, affecting instrument reproducibility. ${ }^{47}$ In the future, a best diagnostic approach may rely in the use of low-cost e-nose device for assessing the presence of PCa in a rapid, non-invasive way, followed by targeted assessment of known volatile biomarkers by GC-MS technology for diagnostic confirmation. The combination of e-nose and GC-MS technologies may provide a powerful tandem diagnostic tool potentially allowing for early non-invasive diagnosis of PCa with high accuracy.

\section{CONCLUSIONS}

In the present study, a comprehensive volatile metabolomic signature of urine from PCa patients was obtained that covered the profile of a large number of volatile carbonyl compounds reported for the first time. A panel of 6 volatile biomarkers was established for $\mathrm{PCa}$ diagnosis, disclosing a good prediction of new $\mathrm{PCa}$ and control samples in an external validation cohort. Indeed, the 6-biomarker panel unveiled higher sensitivity and accuracy compared to serum PSA, as well as higher sensitivity and specificity than fPSA/tPSA. The knowledge gained from the definition of PCa volatile signature in urine samples has the potential to be used in the development of an electronic nose device containing sensing materials tuned for specificity and selectivity, thus improving accuracy. Furthermore, the alterations found in the levels of some metabolites (methylglyoxal, phenylacetaldehyde and hexanal) suggest dysregulations in pyruvate metabolism, glycine, serine and threonine metabolism, phenylalanine metabolism and steroid hormone biosynthesis in prostate carcinogenesis. Nonetheless, the biochemical origin of volatile metabolites remains mostly unknown and further studies focused on the understanding of regulatory mechanisms regarding their release at cellular level are required. In conclusion, our findings strengthen the value of urinary volatilome for PCa diagnosis and disclose a biomarker panel that has potential to be used as an accurate diagnostic tool for this malignancy. Further studies will be performed in order to validate these results in an independent larger cohort.

\section{AUTHOR CONTRIBUTIONS}

A.R.L. was responsible for the execution of experiments, data analysis and preparation of the paper. A.I.A. supported the experimental work and data analysis. J.P. helped with the statistical analysis of the data and contributed to the interpretation of the results. D.B.-S. collected and organised demographic and clinical data from PCa patients. C.J. and R.H. kindly provided urine samples used in the study and gave conceptual advice. M.L.B., P.G.P. and M.C. designed and supervised the study. M.C. also contributed to writing the paper. All authors critically commented on and approved the final submitted version of the paper.

\section{ADDITIONAL INFORMATION}

Supplementary information is available for this paper at https://doi.org/10.1038/ s41416-019-0585-4.

Competing interests: The authors declare no competing interests.

Ethics approval and consent to participate: The study was approved by the Portuguese Oncology Institute of Porto (IPO Porto) Ethics Committee (reference number: $282 \mathrm{R} / 2017$ ) and conducted in accordance with the Declaration of Helsinki. All subjects included in the study provided informed consent at the time of enrolment.

Funding: This work received financial support from the European Union (FEDER funds POCl/01/0145/FEDER/007728) and National Funds (FCT/MEC, Fundação para a Ciência e a Tecnologia and Ministério da Educação e Ciência) under the Partnership Agreement PT2020 UID/MULTI/04378/2013. The study is a result of the project NORTE-01-0145-FEDER-000024, supported by Norte Portugal Regional Operational Programme (NORTE 2020), under the PORTUGAL 2020 Partnership Agreement (DESignBIOtecHealth-New Technologies for three Health Challenges of Modern Societies: Diabetes, Drug Abuse and Kidney Diseases), through the European Regional Development Fund (ERDF). A.R.L. was the recipient of a PhD fellowship from FCT (SFRH/BD/123012/2016) and M.C. acknowledges financial support from FCT through the UID/MULTI/04546/2019 project.

Consent to publish: Not applicable.

Data availability: All data that support the findings of this study are included in this published article and its Supplementary information files.

Note: This work is published under the standard license to publish agreement. After 12 months the work will become freely available and the license terms will switch to a Creative Commons Attribution 4.0 International (CC BY 4.0). 
Publisher's note Springer Nature remains neutral with regard to jurisdictional claims in published maps and institutional affiliations.

\section{REFERENCES}

1. Bray, F., Ferlay, J., Soerjomataram, I., Siegel, R. L., Torre, L. A. \& Jemal, A. Global cancer statistics 2018: GLOBOCAN estimates of incidence and mortality worldwide for 36 cancers in 185 countries. CA Cancer J. Clin. 68, 394-424 (2018).

2. Spur, E. M., Decelle, E. A. \& Cheng, L. L. Metabolomic imaging of prostate cancer with magnetic resonance spectroscopy and mass spectrometry. Eur. J Nucl. Med. Mol Imaging. 40(Suppl 1), S60-S71 (2013).

3. Kearns, J. T. \& Lin, D. W. Improving the specificity of PSA screening with serum and urine markers. Curr. Urol. Rep. 19, 80 (2018).

4. Wolf, A. M., Wender, R. C., Etzioni, R. B., Thompson, I. M., D'Amico, A. V., Volk, R. J. et al. American Cancer Society guideline for the early detection of prostate cancer: update 2010. CA Cancer J. Clin. 60, 70-98 (2010).

5. Kelly, R. S., Vander Heiden, M. G., Giovannucci, E. \& Mucci, L. A. Metabolomic biomarkers of prostate cancer: prediction, diagnosis, progression, prognosis, and Recurrence. Cancer Epidemiol Biomarkers Prev. 25, 887-906 (2016).

6. Huang, Y., Li, Z. Z., Huang, Y. L., Song, H. J. \& Wang, Y. J. Value of free/total prostate-specific antigen ( $f / t$ PSA) ratios for prostate cancer detection in patients with total serum prostate-specific antigen between 4 and $10 \mathrm{ng} / \mathrm{mL}$ : A metaanalysis. Medicine (Baltimore) 97, e0249 (2018).

7. Filella, X., Fernandez-Galan, E., Fernandez Bonifacio, R. \& Foj, L. Emerging biomarkers in the diagnosis of prostate cancer. Pharmgenomics Pers Med. 11, 83-94 (2018).

8. Smith, S., White, P., Redding, J., Ratcliffe, N. M. \& Probert, C. S. J. Application of similarity coefficients to predict disease using volatile organic compounds. IEEE Sens. J. 10, 92-96 (2010).

9. Khalid, T., Aggio, R., White, P., De Lacy Costello, B., Persad, R., Al-Kateb, H. et al. Urinary volatile organic compounds for the detection of prostate cancer. PLoS ONE. 10, e0143283 (2015).

10. Jimenez-Pacheco, A., Salinero-Bachiller, M., Iribar, M. C., Lopez-Luque, A., MijanOrtiz, J. L. \& Peinado, J. M. Furan and p-xylene as candidate biomarkers for prostate cancer. Urol. Oncol. 36, 243e21-e27 (2018).

11. Taverna, G., Tidu, L., Grizzi, F., Torri, V., Mandressi, A., Sardella, P. et al. Olfactory system of highly trained dogs detects prostate cancer in urine samples. J Urol. 193, 1382-1387 (2015).

12. Elliker, K. R., Sommerville, B. A., Broom, D. M., Neal, D. E., Armstrong, S. \& Williams, H. C. Key considerations for the experimental training and evaluation of cancer odour detection dogs: lessons learnt from a double-blind, controlled trial of prostate cancer detection. BMC Urol. 14, 22 (2014).

13. Roine, A., Veskimäe, E., Tuokko, A., Kumpulainen, P., Koskimäki, J., Keinänen Tuomo, A. et al. Detection of prostate cancer by an electronic nose: a proof of principle study. J. Urol. 192, 230-235 (2014).

14. Asimakopoulos, A. D., Del Fabbro, D., Miano, R., Santonico, M., Capuano, R., Pennazza, G. et al. Prostate cancer diagnosis through electronic nose in the urine headspace setting: a pilot study. Prostate Cancer Prostatic Dis. 17, 206 (2014).

15. Bax, C., Taverna, G., Eusebio, L., Sironi, S., Grizzi, F., Guazzoni, G. et al. Innovative diagnostic methods for early prostate cancer detection through urine analysis: a review. Cancers. 10, 123 (2018)

16. Calejo, I., Moreira, N., Araujo, A. M., Carvalho, M., Bastos Mde, L. \& de Pinho, P. G. Optimisation and validation of a HS-SPME-GC-IT/MS method for analysis of carbonyl volatile compounds as biomarkers in human urine: application in a pilot study to discriminate individuals with smoking habits. Talanta. 148, 486-493 (2016).

17. Monteiro, M., Carvalho, M., Henrique, R., Jeronimo, C., Moreira, N., de Lourdes Bastos, M. et al. Analysis of volatile human urinary metabolome by solid-phase microextraction in combination with gas chromatography-mass spectrometry for biomarker discovery: application in a pilot study to discriminate patients with renal cell carcinoma. Eur. J. Cancer 50, 1993-2002 (2014)

18. Pluskal, T., Castillo, S., Villar-Briones, A. \& Oresic, M. MZmine 2: modular framework for processing, visualizing, and analyzing mass spectrometry-based molecular profile data. BMC Bioinformatics 11, 395 (2010).

19. Xi, B., Gu, H., Baniasadi, H. \& Raftery, D. Statistical analysis and modeling of mass spectrometry-based metabolomics data. Methods Mol. Biol. 1198, 333-353 (2014).

20. Chong, J., Soufan, O., Li, C., Caraus, I., Li, S., Bourque, G. et al. MetaboAnalyst 4.0: towards more transparent and integrative metabolomics analysis. Nucleic Acids Res. 46(W1), W486-W494 (2018).

21. Fawcett, T. An introduction to ROC analysis. Pattern Recogn. Lett. 27, 861-874 (2006).

22. Berben, L., Sereika, S. M. \& Engberg, S. Effect size estimation: methods and examples. Int. J. Nurs Stud. 49, 1039-1047 (2012).
23. Aickin, M. \& Gensler, H. Adjusting for multiple testing when reporting research results: the Bonferroni vs Holm methods. American journal of public health. $\mathbf{8 6}$ 726-728 (1996).

24. R Core Team. R: A Language and Environment for Statistical Computing. ( $R$ Foundation for Statistical Computing, Vienna, Austria, 2014).

25. Dimakakos, A., Armakolas, A. \& Koutsilieris, M. Novel tools for prostate cancer prognosis, diagnosis, and follow-up. Biomed Res Int. 2014, 890697 (2014).

26. Marchand C.R., Farshidfar F., Rattner J. \& Bathe O.F. A framework for development of useful metabolomic biomarkers and their effective knowledge translation. Metabolites 8, 59 (2018).

27. Hussain A., Semeano A. T. S., Palma S., Pina A. S., Almeida J., Medrado B. F. et al. TunaBle Gas Sensing Gels By Cooperative Assembly. Adv. Funct. Mater. 27 1700803 (2017).

28. Haick, H., Broza, Y. Y., Mochalski, P. \& Ruzsanyi, V. Amann A. Assessment, origin and implementation of breath volatile cancer markers. Chem. Soc. Rev. 43, 1423-1449 (2014).

29. Wishart, D. S., Feunang, Y. D., Marcu, A., Guo, A. C., Liang, K., Vázquez-Fresno, R. et al. HMDB 4.0: the human metabolome database for 2018. Nucleic Acids Res. 46 (D1), D608-D617 (2018)

30. Zhang, Y., Gao, G., Liu, H., Fu, H., Fan, J., Wang, K. et al. Identification of volatile biomarkers of gastric cancer cells and ultrasensitive electrochemical detection based on sensing interface of Au-Ag alloy coated MWCNTs. Theranostics. 4 154-162 (2014).

31. Taware, R., Taunk, K., Pereira, J. A. M., Dhakne, R., Kannan, N., Soneji, D. et al. Investigation of urinary volatomic alterations in head and neck cancer: a noninvasive approach towards diagnosis and prognosis. Metabolomics. 13, 111 (2017).

32. Khandrika, L., Kumar, B., Koul, S., Maroni, P. \& Koul, H. K. Oxidative stress in prostate cancer. Cancer Lett. 282, 125-136 (2009).

33. Oh, B., Figtree, G., Costa, D., Eade, T., Hruby, G., Lim, S. et al. Oxidative stress in prostate cancer patients: a systematic review of case control studies. Prostate int. 4, 71-87 (2016).

34. Nogueira, M. N., Aquino, S. G., Rossa Junior, C. \& Spolidorio, D. M. Terpinen-4-ol and alpha-terpineol (tea tree oil components) inhibit the production of IL-1beta, IL-6 and IL-10 on human macrophages. Inflamm Res. 63, 769-778 (2014).

35. Ryu, N. H., Park, K. R., Kim, S. M., Yun, H. M., Nam, D., Lee, S. G. et al. A hexane fraction of guava Leaves (Psidium guajava L.) induces anticancer activity by suppressing AKT/mammalian target of rapamycin/ribosomal p70 S6 kinase in human prostate cancer cells. J. Med. Food 15, 231-241 (2012).

36. Zhao, Y., Chen, R., Wang, Y., Qing, C., Wang, W. \& Yang, Y. In vitro and in vivo efficacy studies of lavender angustifolia essential oil and its active constituents on the proliferation of human prostate cancer. Integr. Cancer Ther. 16, 215-226 (2017).

37. Muzio, G., Maggiora, M., Paiuzzi, E., Oraldi, M. \& Canuto, R. A. Aldehyde dehydrogenases and cell proliferation. Free Radic. Biol. Med. 52, 735-746 (2012).

38. Yan, J., De Melo, J., Cutz, J. C., Aziz, T. \& Tang, D. Aldehyde dehydrogenase 3A1 associates with prostate tumorigenesis. Br. J. Cancer 110, 2593-2603 (2014)

39. Li, D. \& Ellis, E. M. Aldo-keto reductase 7A5 (AKR7A5) attenuates oxidative stress and reactive aldehyde toxicity in V79-4 cells. Toxicol. In Vitro. 28, 707-714 (2014).

40. Lima A. R., Araujo A. M., Pinto J., Jeronimo C., Henrique R., Bastos M. L. et al. GCMS-based endometabolome analysis differentiates prostate cancer from normal prostate cells. Metabolites. 19, pii: E23 (2018).

41. White, H. \& Venkatesh, B. Clinical review: ketones and brain injury. Crit. Care. 15 219 (2011).

42. Serrano, M., Gallego, M. \& Silva, M. Analysis of endogenous aldehydes in human urine by static headspace gas chromatography-mass spectrometry. J. Chromatogr. A. 1437, 241-246 (2016).

43. Sadowska-Bartosz, I. \& Bartosz, G. Effect of glycation inhibitors on aging and agerelated diseases. Mech. Ageing Dev. 160, 1-18 (2016).

44. Lee, D. K., Na, E., Park, S., Park, J. H., Lim, J. \& Kwon, S. W. In vitro tracking of intracellular metabolism-derived cancer volatiles via isotope labeling. ACS Cent. Sci. 4, 1037-1044 (2018).

45. Wilson, A. D. \& Baietto, M. Advances in electronic-nose technologies developed for biomedical applications. Sensors (Basel). 11,1105-1176 (2011).

46. Wilson, A. D. Advances in electronic-nose technologies for the detection of volatile biomarker metabolites in the human breath. Metabolites. 5, 140-163 (2015).

47. Capelli L., Taverna G., Bellini A., Eusebio L., Buffi N., Lazzeri M. et al. Application and uses of electronic noses for clinical diagnosis on urine samples: a review. Sensors (Basel). 16, pii: E1708 (2016).

48. Bianchi, F., Riboni, N., Carbognani, P., Gnetti, L., Dalcanale, E., Ampollini, L. et al. Solid-phase microextraction coupled to gas chromatography-mass spectrometry followed by multivariate data analysis for the identification of volatile organic compounds as possible biomarkers in lung cancer tissues. J. Pharm. Biomed. Anal. 146, 329-333 (2017)

49. Taunk, K., Taware, R., More, T. H., Porto-Figueira, P., Pereira, J. A. M., Mohapatra, R. et al. A non-invasive approach to explore the discriminatory potential of the 
Identification of a biomarker panel for improvement of prostate cancer...

AR Lima et al.

urinary volatilome of invasive ductal carcinoma of the breast. RSC Advances 8, 25040-25050 (2018)

50. Miyata, T., Inagi, R., Asahi, K., Yamada, Y., Horie, K., Sakai, H. et al. Generation of protein carbonyls by glycoxidation and lipoxidation reactions with autoxidation products of ascorbic acid and polyunsaturated fatty acids. FEBS Lett. 437, 24-28 (1998).

51. Liu, X. Y., Yang, Z. H., Pan, X. J., Zhu, M. X. \& Xie, J. P. Crotonaldehyde induces oxidative stress and caspase-dependent apoptosis in human bronchial epithelial cells. Toxicol Lett. 195, 90-98 (2010).
52. Voulgaridou, G. P., Anestopoulos, I., Franco, R., Panayiotidis, M. I. \& Pappa, A. DNA damage induced by endogenous aldehydes: current state of knowledge. Mutat Res. 711, 13-27 (2011).

53. Garner, C. E., Smith, S., de Lacy Costello, B., White, P., Spencer, R., Probert, C. S. et al. Volatile organic compounds from feces and their potential for diagnosis of gastrointestinal disease. FASEB J. 21, 1675-1688 (2007).

54. Viant, M. R., Kurland, I. J., Jones, M. R. \& Dunn, W. B. How close are we to complete annotation of metabolomes? Curr. Opin. Chem. Biol. 36, 64-69 (2017). 